\title{
The Role of $P \tau$ in the Photothermoelectric Effect and in Photoredox Catalysis Reactions
}

\author{
Giovanna Scarel \\ Department of Physics and Astronomy, James Madison University, Harrisonburg, VA, USA \\ Email: scarelgx@jmu.edu
}

How to cite this paper: Scarel, G. (2019) The Role of $P \tau$ in the Photothermoelectric Effect and in Photoredox Catalysis Reactions. World Journal of Condensed Matter Physics, 9, 91-101.

https://doi.org/10.4236/wjcmp.2019.94007

Received: August 29, 2019

Accepted: September 17, 2019

Published: September 20, 2019

Copyright $\odot 2019$ by author(s) and Scientific Research Publishing Inc. This work is licensed under the Creative Commons Attribution International License (CC BY 4.0).

http://creativecommons.org/licenses/by/4.0/

\begin{abstract}
Context and Background: Recent research has shown that the amount of energy conserved in light-matter interaction is given by the product of light's power $P$ times its period $\tau$, i.e. $P \tau$. To date, evidences of the validity of such finding are restricted to the interaction of light with capacitors, infrared spectroscopy, and vision in vertebrates. Motivation: In this article, we want to explore the validity of the role of $P \tau$ in a broader range of phenomena. Hypothesis: We assume that the photothermoelectric (PTE) effect and photoredox catalysis reactions (PCRs) are manifestations of light-matter interaction and therefore have $P \tau$ conserved in the process. Method: We take the data published in two articles, one on the PTE effect and the other on PCRs and revisit the data analysis of the authors of the original articles considering $P \tau$ as the energy conserved. Results: In the case of the PTE effect, we unveil that the size of the light's beam cross-sectional area impinging on the photodetectors plays a major role in defining the performance of the photodetectors. With our analysis, the photodetector responsivities actually turn out to be higher than those reported in the original article. In the case of the PCRs, we find that the magnitude of $P \tau$ involved in successful PCRs is independent of the type of light used, whether near-infrared or blue. In addition, the involvement of $P \tau$ in the description of PCRs helps to clarify the role of the law of conservation of energy, which was neglected by the authors of the original article. Conclusions: From this study, we infer that the hypothesis that $P \tau$ represents the amount of energy conserved in light-matter interaction is valid and general, useful to measure device performance, and predict alternative processes to achieve desired outcomes.
\end{abstract}

\section{Keywords}

Light-Matter Interaction, Photothermoelectric Effect, Photoredox Reactions 


\section{Introduction}

Recently, experimental evidences regarding the interaction of visible and infrared (IR) light with capacitors [1] highlighted that the experimental results are explained by acknowledging that, in light-matter interaction, the total energy, or energy conserved, is given by the product of light's power $P$ times its period $\tau$, i.e. $P \tau$ [1]. No other research group to date achieved a similar conclusion.

With our own experiments, we have achieved the evidence that $P \tau$ as the energy conserved in light-matter interaction is capable of precisely explaining the numerical outcome of voltage from capacitors illuminated by IR light. This result led us to the inquiry whether the validity of $P \tau$ might be extended to phenomena other than the interaction of light with capacitors. Since there exist numerous experiments based on light-matter interaction published in the scientific literature, we decided to make use of them to test the hypothesis on $P \tau$. As we find that the energy $P \tau$ efficiently captures the orders of magnitude of the outcomes from both our experiments and those of other authors, we conclude that the concept of photon is not necessary in the analysis of light-matter phenomena. Our review of the literature on light-matter interaction, specifically on the photothermoelectric (PTE) effect [2] and the photoredox catalysis reactions (PCRs) [3], is important to assess the primary role of $P \tau$ in light-matter interaction and possibly to pave the way to new results in various areas of science and technology involving electromagnetic waves. In particular, assessing that $P \tau$ is the amount of energy conserved in light-matter interaction impacts: 1 ) the quantitative analysis of light-matter interaction; 2) the determination of the effectiveness of the PTE effect, the PCRs and other mechanisms; and 3) the establishment of the conditions required to obtain similar effects with different wavelengths.

\section{Method}

We assess the ability of $P \tau$ to capture the amount of energy conserved in lightmatter interaction by revisiting the analysis to experiments on light-matter interaction performed by other authors. We have chosen two experiments, one related to the PTE effect [2] and the other to PCRs [3]. We use data published in the corresponding articles, and perform our own data analysis assuming $P \tau$ as the energy conserved. We then compare our conclusions with those achieved by the authors of the original articles.

\section{The Photothermoelectric Effect}

The paper by $\mathrm{Lu}$ et al. [2] describes fabrication and performance of reduced $\mathrm{SrTiO}_{3}$ (r-STO) based PTE photodetectors, producing high photovoltage responsivity $\left(\pi_{V}\right.$, i.e. the ratio between the photovoltage produced and the power of the impinging light) and broadband spectral response from $325 \mathrm{~nm}$ to $10.57 \mu \mathrm{m}$. The authors ascribe the enhanced responsivity to the existence of a $\mathrm{Ti}-\mathrm{O}$ phonon mode in the long-wavelength infrared region (LWIR), in agreement with findings in 
the interaction of IR light with thin films [4].

The data provided by $\mathrm{Lu}$ et al. [2] do not clearly correlate the photovoltage $\Delta V$ and responsivity $\pi_{V}$ produced by the r-STO PTE photodetectors to the characteristics, e.g. beam size and power $P$, of the impinging light. This lack of correlation in the trends between light's characteristics and r-STO PTE photodetector's outcome ( $\Delta V$ and $\pi_{V}$ ) shadows the role of device size on the enhancement of the PTE effect ascribed to the Ti-O phonon modes. Here, based on the hypothesis that the product of light's power $P$ times its period $\tau$, i.e. $P \tau$ [1], is the energy conserved in light-matter interaction, we propose that a trend exists between light's characteristics and r-STO PTE photodetector outcome. Accordingly, we review the data published by $\mathrm{Lu}$ et al. [2]. In addition, we treat the r-STO PTE photodetector as a capacitor with capacitance $C$, similarly to the treatment given to thermoelectric devices in [1] and [5] due their multi-layer structure alternating conducting and non-conducting elements.

The main characteristics of the laser beam used by Lu et al. [2] to illuminate the r-STO PTE photodetectors, the produced photovoltage $\Delta V$, and the resulting responsivity $\pi_{V}$, are collected in Table 1 . For the illumination with $\lambda=1550 \mathrm{~nm}$, we chose to display the values of $\Delta V$ and $\pi_{V}$ generated when $P=100 \mathrm{~mW}$ and displayed in Figure 6(b) of [2].

With the assumptions that $P \tau$ is the total energy in light-matter interaction, and treating the r-STO PTE photodetector as a capacitor with capacitance $C$, we use the equation $P \tau \cong \frac{1}{2} C \Delta V^{2}$ to evaluate the energy transferred from the light's beam to the photodetectors such that a photovoltage $\Delta V$ is produced. This equation agrees with Equations (2a) and (2b) in [1], and is an approximated expression because here we neglect a thermal component which, in order to be significant, needs to be of the same order of magnitude of $P \tau$ [1]. With the

Table 1. Characteristics of the impinging light (wavelength $\lambda$, period $\tau$, power $P$, and spot radius $r$ ) and r-STO PTE photodetector outcome (photovoltage $\Delta V$ and responsivity $\pi_{V}$, i.e. the ratio between photovoltage produced and power of the impinging light). The data are taken from Lu et al. [2]. The spot radius is the radius of the beam with circular cross-section and diameter equal to the beam size given by $\mathrm{Lu}$ et al. [2]. For the illumination with $\lambda=1550 \mathrm{~nm}$ we display the values of photovoltage $\Delta V$ and responsivity $\pi_{V}$ generated when $P=100 \mathrm{~mW}$ and displayed in Figure 6(b) of [2]. The capacitance of the r-STO PTE photodetectors studied by Lu et al. [2] is estimated as $C=\frac{2 P \tau}{\Delta V^{2}}$. The average $C$ value of these devices is $(1.93 \pm 1.65) \mathrm{pF}$.

\begin{tabular}{ccccccc}
\hline$\lambda(\mathrm{nm})$ & $\tau(\mathrm{fs})$ & $P(\mathrm{~mW})$ & $r(\mu \mathrm{m})$ & $\Delta V(\mathrm{mV})$ & $\pi_{V}(\mathrm{~V} / \mathrm{W})$ & $C(\mathrm{pF})$ \\
\hline 325 & 1.08 & 2.3 & 2.5 & 2.6 & 1.13 & 0.74 \\
532 & 1.77 & 3.4 & 3 & 2.9 & 0.85 & 1.43 \\
785 & 2.62 & 5.1 & 5 & 3.1 & 0.61 & 2.78 \\
1550 & 5.17 & 100 & 100 & 57 & 0.57 & 0.32 \\
10,570 & 35.23 & 11.6 & 15 & 11 & 0.95 & 4.36 \\
\hline
\end{tabular}


expression for $P \tau$ given above, and assuming the beam sizes given in [2] to be the diameter of the light's beam with circular cross-section of radius $r$, we estimate the value of the capacitance $C=\frac{2 P \tau}{\Delta V^{2}}$ of the photodetectors studied by Lu et al. [2]. The values of $C$ are reported in the last column of Table 1 . It is interesting to note that the $C$ values obtained with the data provided in [2] are of the same order of magnitude for the five different types of illumination, and have an average value of $(1.93 \pm 1.65) \mathrm{pF}$.

To achieve our goal of finding a correlation between light's characteristics and r-STO PTE photodetector outcome in the experiments performed by $\mathrm{Lu}$ et al. [2], we suppose that, at all wavelengths, the beam size is the same, e.g. $10 \mu \mathrm{m}$. We then rescale on a cross-sectional area $A=\pi r^{2}$ with spot radius $r=5 \mu \mathrm{m}$ the values of $C$ of the r-STO PTE photodetectors used by Lu et al. [2]. To this end, we exploit the direct proportionality between $C$ and $A$ given by $C=\kappa \varepsilon_{0} \frac{A}{d}$, where $\varepsilon_{0}=8.854 \times 10^{-12} \mathrm{~F} / \mathrm{m}$ is the permittivity in vacuum, $\kappa$ is the dielectric constant varying between 1 to $10^{3}, A=\pi r^{2}$ is the cross-sectional area of the beam hitting the photodetector, and $d$ is the distance between the electrodes, or plates, of the capacitors. The rescaled capacitances are reported in Table 2 . We observe that the average $C$ value is $(2.04 \pm 1.47) \mathrm{pF}$, similar within the errors to the average capacitance found from the data in Table 1 . We also observe that the capacitance of the photodetector used for the illumination with the beam at $\lambda=1550 \mathrm{~nm}$ is three orders of magnitude lower than that of the photodetectors used with the other four wavelengths considered. With the values of the rescaled capacitances $C$ and using $\Delta V=\sqrt{\frac{2 P \tau}{C}}$, we rescale also the photovoltage $\Delta V$

Table 2. Here we consider the characteristics of the impinging light (wavelength $\lambda$, period $\tau$, and power $P$ ) to be the same as in Lu et al. [2]. The spot radius, however, is assumed to always be $r=5 \mu \mathrm{m}$. The capacitances of the r-STO PTE photodetectors are then rescaled on a cross-sectional area $A=\pi r^{2}$ with $r=5 \mu \mathrm{m}$ exploiting the direct proportionality between $C$ and $A$ given by $C=\kappa \varepsilon_{0} \frac{A}{d}$, where $\varepsilon_{0}=8.854 \times 10^{-12} \mathrm{~F} / \mathrm{m}$ is the permittivity in vacuum, $\kappa$ is the dielectric constant varying between 1 to $10^{3}$, and $d$ the distance between the electrodes. The average $C$ value is $(2.04 \pm 1.47) \mathrm{pF}$. The photovoltage is calculated as $\Delta V=\sqrt{\frac{2 P \tau}{C}}$, and used to estimate the responsivity $\pi_{V}$.

\begin{tabular}{ccccccc}
\hline$\lambda(\mathrm{nm})$ & $\tau(\mathrm{fs})$ & $P(\mathrm{~mW})$ & $r(\mu \mathrm{m})$ & $\Delta V(\mathrm{mV})$ & $\pi_{V}(\mathrm{~V} / \mathrm{W})$ & $C(\mathrm{pF})$ \\
\hline 325 & 1.08 & 2.3 & $\mathbf{5}$ & 1.30 & 0.57 & 2.95 \\
532 & 1.77 & 3.4 & $\mathbf{5}$ & 1.74 & 0.51 & 3.97 \\
785 & 2.62 & 5.1 & $\mathbf{5}$ & 3.10 & 0.61 & 2.78 \\
1550 & 5.17 & 100 & $\mathbf{5}$ & 113.24 & 1.14 & 0.0008 \\
10,570 & 35.23 & 11.6 & $\mathbf{5}$ & 33.01 & 2.85 & 0.48 \\
\hline
\end{tabular}


and responsivity $\pi_{V}$, both of which are reported in Table 2 . We observe that now the responsivities increase with the light's wavelength $\lambda$ (or with light's period $\tau$ ), as predicted in [1]. More interestingly, the responsivities at the larger wavelengths, $1550 \mathrm{~nm}$ and $10.57 \mu \mathrm{m}(1.14 \mathrm{~V} / \mathrm{W}$ and $2.85 \mathrm{~V} / \mathrm{W}$, respectively), are larger than the highest responsivity reported in [2] (1.13 V/W) and in [1] $(0.2 \mathrm{~V} / \mathrm{W})$. Additionally, we notice that the responsivity $\pi_{V}$ at $532 \mathrm{~nm}$ is 0.51 $\mathrm{V} / \mathrm{W}$, smaller than the one of $0.57 \mathrm{~V} / \mathrm{W}$ at $325 \mathrm{~nm}$. We ascribe this finding to the fact that we neglected the thermal component in estimating $\Delta V$ at the lower wavelengths, where the temperature difference between the plates of the capacitors becomes significant according to findings in [1].

We can further rescale the photovoltage $\Delta V$ and responsivity $\pi_{V}$ by assuming all r-STO PTE photodetectors to have a spot radius $r=5 \mu \mathrm{m}$ and a capacitance $C=2.04 \pm 1.47 \mathrm{pF}$, the average value found from the data in Table 2 . This assumption is reasonable because capacitance is a device's property; therefore, if all the devices are prepared in the same way they should have the same $C$. The newly rescaled values for $\Delta V$ and $\pi_{V}$ are reported in Table 3. We notice that this time the responsivities increase with the light's wavelength $\lambda$ (or with light's period $\tau$ ), as predicted in [1]. There are very small differences among the $\pi_{V}$ values at $\lambda=325,532$, and $785 \mathrm{~nm}$. The responsivity at $10.57 \mu \mathrm{m}$ is $1.72 \mathrm{~V} / \mathrm{W}$, again larger than the highest responsivity reported in [2] $(1.13 \mathrm{~V} / \mathrm{W})$ and in [1] $(0.2 \mathrm{~V} / \mathrm{W})$. However, the responsivity $\pi_{V}$ at $\lambda=1550 \mathrm{~nm}$ is anomalously small $(0.22 \mu \mathrm{m})$. The values of the responsivities versus light's period $\tau$ are reported in Figure 1, where the empty stars depict the values from Table 2 and the small empty circles report those from Table 3.

In Table 2 and Table 3 we observed three anomalous results with the photodetector used for the illumination with the beam at $\lambda=1550 \mathrm{~nm}$ with spot radius $r=5 \mu \mathrm{m}$. The first is that the capacitance is three orders of magnitude lower than that for the devices used at all other wavelengths (Table 2). The second is that the responsivity $\pi_{V}$ for the device with $C=2.04 \mathrm{pF}$ is anomalously small $(0.22 \mathrm{~V} / \mathrm{W}$, as in Table 3$)$. The third is that even the intensity used in the case of $\lambda=1550 \mathrm{~nm}$ is anomalously small, as shown in Table 4 . We suggest that these anomalies result from sample preparation.

In conclusion, we have found that the use of $P \tau$ as the total energy, or the energy conserved, in light's interaction with an r-STO PTE photodetector enables highlighting the trends in the responsivity as a function of light's wavelength or period. First, the size of the light's beam cross-sectional area impinging on the r-STO PTE photodetectors plays a major role in defining the performance of the photodetectors. Then, in agreement with [1], the responsivities increase with increasing light's wavelength or period. This conclusion is supported by the result, obtained with a $45 \mathrm{pF}$ thermoelectric device, that with microwaves, i.e. with wavelength much larger than that of visible light, the responsivity is $\pi_{V}=7 \times 10^{2} \mathrm{~V} / \mathrm{W}$ [5]. Finally, we explain the anomaly for the device illuminated with light at $\lambda=1550 \mathrm{~nm}$ as consequence of the sample preparation. 
Table 3. Here we consider the characteristics of the impinging light (wavelength $\lambda$, period $\tau$, and power $P$ ) to be the same as in Lu et al. [2]. The spot radius is always $r=5 \mu \mathrm{m}$. The capacitances of the r-STO PTE photodetectors are assumed to be all equal to the average value of $(2.04 \pm 1.47) \mathrm{pF}$ found in Table 2 . The photovoltage is calculated as $\Delta V=\sqrt{\frac{2 P \tau}{C}}$, and used to estimate the responsivity $\pi_{V}$.

\begin{tabular}{ccccccc}
\hline$\lambda(\mathrm{nm})$ & $\tau(\mathrm{fs})$ & $P(\mathrm{~mW})$ & $r(\mu \mathrm{m})$ & $\Delta V(\mathrm{mV})$ & $\pi_{V}(\mathrm{~V} / \mathrm{W})$ & $C(\mathrm{pF})$ \\
\hline 325 & 1.08 & 2.3 & $\mathbf{5}$ & 1.56 & 0.68 & 2.04 \\
532 & 1.77 & 3.4 & $\mathbf{5}$ & 2.43 & 0.71 & 2.04 \\
785 & 2.62 & 5.1 & $\mathbf{5}$ & 3.62 & 0.71 & 2.04 \\
1550 & 5.17 & 100 & $\mathbf{5}$ & 22.51 & 0.22 & 2.04 \\
10,570 & 35.23 & 11.6 & $\mathbf{5}$ & 20.02 & 1.72 & 2.04 \\
\hline
\end{tabular}

Table 4. Intensity (power over area) of the impinging light at the various wavelenghts used by Lu et al. to illuminate their r-STO PTE photodetectors [2].

\begin{tabular}{cc}
\hline Wavelength $\lambda(\mathrm{nm})$ & Intensity $\mathrm{I}\left(10^{5} \mathrm{~W} / \mathrm{cm}^{2}\right)$ \\
\hline 325 & 0.117 \\
532 & 0.120 \\
785 & 0.065 \\
1550 & 0.003 \\
10,570 & 0.016 \\
\hline
\end{tabular}

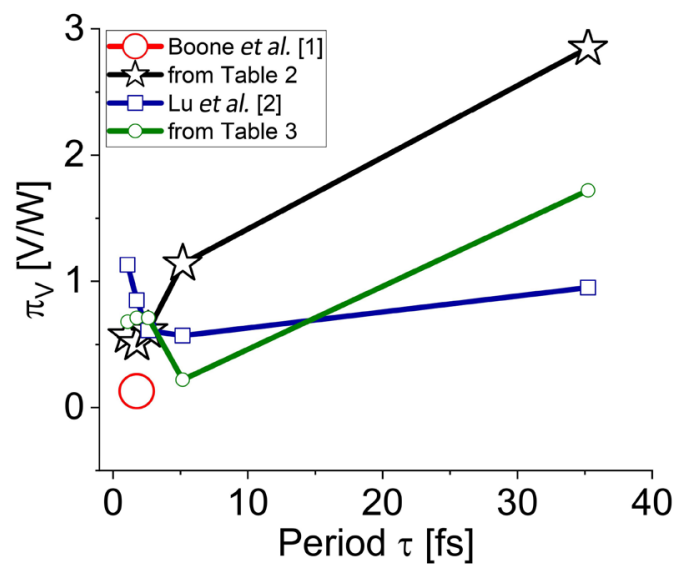

Figure 1. Values of the responsivities $\pi_{V}$ versus light's period $\tau$. The data are taken from Boone et al. [1] (large empty circles), and from Lu et al. [2] (empty squares). The empty stars depict the responsivity $\pi_{V}$ values taken from Table 2, where each value of the capacitance $C$ and photovoltage $\Delta V$ of the r-STO PTE photodetectors was rescaled assuming a spot size of $r=5 \mu \mathrm{m}$. The small empty circles represent the $\pi_{V}$ values taken from Table 3 , where all photodetectors are illuminated on an area that corresponds to the light's beam cross-section with radius $r=5 \mu \mathrm{m}$, and all photodetectors are assumed to have capacitance $C=2.04 \mathrm{pF}$, the average value found in Table 2 . The photovoltages produced by the photodetectors are listed in Table 3. 


\section{The Photoredox Catalysis Reactions}

The article by Ravetz et al. [3] exploits the mechanism of triplet fusion upconversion in PCRs. Triplet fusion upconversion consists of the transformation of low energy photons into high energy ones. Specifically, starting from near infrared (NIR) photons, Ravetz et al. [3] access photons in the orange to blue light interval to enable several photoredox catalysis reactions (PCRs). Triplet fusion upconversion involves various steps of excitation and decay processes. The main goal of the process is to create a higher-energy singlet exciton which, then, decays giving off a high-energy photon [3]. However, from [3] it is not clear how conservation of energy is satisfied in the upconversion from NIR to blue light. More specifically, one NIR photon with wavelength $\lambda=730 \mathrm{~nm}$ and frequency $v=0.41 \times 10^{15} \mathrm{~Hz}$ has energy $h v=2.72 \times 10^{-19} \mathrm{~J}$, while a blue photon with $\lambda=450 \mathrm{~nm}$ and $v=0.67 \times 10^{15} \mathrm{~Hz}$ has energy $h v=4.44 \times 10^{-19} \mathrm{~J}$. Here $h=6.67 \times 10^{-34} \mathrm{~J} \cdot \mathrm{s}$ is Planck's constant. The difference in energy between the blue and NIR photons is $\Delta E_{\text {blue-NIR }}=1.72 \times 10^{-19} \mathrm{~J}$. Ravetz et al. [3] do not specify what mechanism supplies $\Delta E_{\text {blue-NIR }}$ within the upconversion process.

To address the problem of finding the source of $\Delta E_{\text {blue-NIR }}$ satisfying the law of conservation of energy, we assume that, in light-matter interaction, the total energy, or the energy conserved, is given by the product of light's power $P$ times its period $\tau$, i.e. $P \tau$ [1], where $\tau$ is $\frac{1}{v}$. To investigate the effectiveness of $P \tau$ as the total energy conserved in PCRs, we collect the information available on light characteristics ( $\lambda, \tau$ and $P$ ) of the light sources used by Ravetz et al. [3], and calculate the corresponding $P \tau$. Table 5 summarizes the collected information, including the type of source (laser diode or light emitting diode-LED) and the specific location in [3] in which the information was found.

The first two rows in Table 5 describe the characteristics of the laser light used by Ravetz et al. [3] to trigger the reaction forming a crosslinked PMMA gel. The reaction is successful when excited with both NIR and blue light transferred to the reactants through air and water. We notice that the values of the used $P \tau$ for the NIR and blue light are with good approximation of the same order of magnitude: $0.1 \mathrm{fJ}$ for the NIR light and $2.4 \mathrm{fJ}$ for the blue light. There is a gap of $2.3 \mathrm{fJ}$ between the $P \tau$ values of the NIR and the blue light. The gap can be observed in Figure 2(a) where it is clear that the $P \tau$ area of the blue light is larger than the $P \tau$ area of the NIR light. We ascribe this gap to the uncertainty of the exact value of $P$ capable of promoting with maximum efficiency the freestanding gel-forming PCRs through the NIR and the blue light sources.

The third and fourth rows in Table 5 report the characteristics of the LEDs used by Ravetz et al. [3] to trigger the same crosslinked PMMA gel-forming reaction described above. In this case, according to the data reported in Table S1 of [3], the authors aimed at performing the reaction by constraining the light to pass through three sheets of white printer paper. The reaction turned out to be successful only using NIR light. It is interesting to note that Ravetz et al. [3] choose the light characteristics of the LED lamp such that the $P \tau$ s of both the NIR and the blue 
light are nearly the same: $37.5 \mathrm{fJ}$ for the NIR light and $53.5 \mathrm{fJ}$ for the blue light. The good match can be observed in Figure 2(b) where we observe that the $P \tau$ area of the blue light is of the same order of magnitude of the $P \tau$ area of the NIR light. Ravetz et al. [3] ascribe the lack of success of the blue light in producing a successful gel-forming reaction to the insufficient penetration depth of the blue light in the three sheets of white printer paper. We assume however that, with the given values of $P$ and $\tau$, the reaction would have been successful with both the NIR and the blue light if the reactants would have been illuminated through air and water.

Table 5. Type of light source, wavelength $(\lambda)$, period $(\tau)$, and power $(P)$ used by Ravetz et al. [3] in promoting the various photoredox catalysis reactions (PCRs) with either near infrared (NIR) or blue light. For each case, we report the values of $P \tau$, the energy conserved in light-matter interaction. The last column indicates the location in [3] describing the various PCRs discussed here.

\begin{tabular}{cccccc}
\hline Type & $\lambda(\mathrm{nm})$ & $\tau(\mathrm{fs})$ & $P(\mathrm{~mW})$ & $P \tau(\mathrm{fJ})$ & Location within [3] \\
\hline Laser diode & 730 & 2.44 & 40 & 0.1 & Table S1 \\
Laser diode & 450 & 1.5 & 1600 & 2.4 & Table S1 \\
LED & $\sim 750$ & 2.5 & 15,000 & 37.5 & Table S1 \\
LED & $\sim 460$ & 1.53 & 35,000 & 53.55 & Table S1 \\
Laser diode & 730 & 2.44 & 16.8 & 0.041 & Figure S9 \\
Laser-diode & 450 & 1.5 & 37.2 & 0.056 & Figure S9 \\
\hline
\end{tabular}
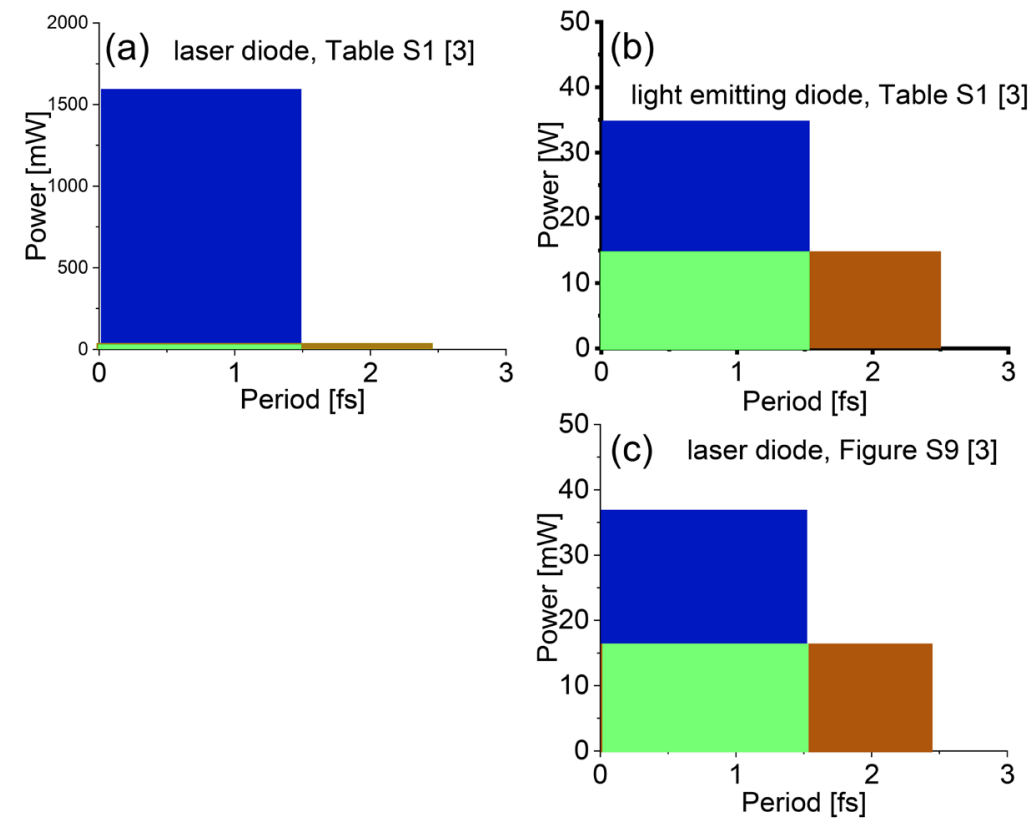

Figure 2. Representation of $P \tau$ for the various illuminations presented by Ravetz et al. [3]: (a) laser diode (Table S1), (b) light emitting diode (Table S1), and (c) laser diode (Figure S9). The blue area is the $P \tau$ for the blue light illumination, the dark-orange area is the $P \tau$ for the near-infrared illumination, and the green area is the overlap between the two areas. 
Finally, the fifth and sixth rows in Table 5 illustrate the characteristics of the laser light that Ravetz et al. determined to be successful in promoting the reactions reported in Figure 2 of [3]. The reported values of $P$ actually represent the power absorbed by the reactants to activate those reactions. In the successful cases, the values of $P \tau$, reported in Table 5, are $0.041 \mathrm{fJ}$ for the NIR light and $0.056 \mathrm{fJ}$ for the blue light. The agreement between the values of $P \tau$ for the two types of light is excellent, as can be observed in Figure 2(c) where the $P \tau$ area of the blue light is of the same order of magnitude of the $P \tau$ area of the NIR light. Therefore, from the results in Table 5, we conclude that in all the successful PCRs the amount of energy conserved in the transfer of energy from light to reactants is $P \tau$, independently of the type of light used. This finding enables us to capture the role of the law of conservation of energy in the PCRs presented by Ravetz et al. [3].

The actual values of $P \tau$ calculated in reviewing the analysis by Ravetz et al. deserve some comment. Let us pick from Table 5 the $P \tau=0.056 \mathrm{fJ}$ for, e.g., the reaction reported in Figure 2(d) of [3] describing the intramolecular [2+2] cyclization of enones through a prototypical $\left[\mathrm{Ru}(\mathrm{bpy})_{3}\right]^{2+}$-catalyzed reaction and blue light. First, if the whole amount of $0.056 \mathrm{fJ}$ is needed for the activation of one enone molecule, then we estimate that $\approx 80 \mathrm{Mcal} / \mathrm{mole}$ would be required for enones. This is an enormous amount of energy compared to the standard reaction activation energies, which are of the order of about tens of kcal/mole! Perhaps a mechanism exists that reduces the energy of the absorbed blue light by, e.g.: 1) emission of light at a different wavelength, as mentioned in [3], 2) reflection of light, and, possibly, 3) dissipation of light's energy in the form of thermal energy. Second, if the energy $h v=4.44 \times 10^{-19} \mathrm{~J}$ of an absorbed blue photon is needed to activate one enone, then $64 \mathrm{kcal} / \mathrm{mole}$ would be required for enones. This quantity is three orders of magnitude smaller than the corresponding amount in terms of $P \tau$, i.e. $\approx 80 \mathrm{Mcal} / \mathrm{mole}$, but closer to the standard reaction activation energies. Thus, if the energy $h v$ of an absorbed blue photon is needed to activate one enone, then there would be almost no fraction of the absorbed blue light available for emission, reflection and dissipation, which, however, are experimentally observed [3].

To summarize, we use the characteristics of the light used to activate PCRs by Ravetz et al. [3] to find the values of $P \tau$, i.e. the product of light's power $P$ times its period $\tau$. For each specific successful reaction considered by Ravetz et al. [3], we find that the values of $P \tau$ are nearly the same whether the reactions are activated with NIR or blue light. The different values of the power at the different wavelengths considered (either blue or NIR) enable reaching the same $P \tau$. Other recent articles indicate the crucial role played by power $P$ in determining the success or failure of photo-excited chemical reactions [6]. This finding supports the hypothesis that $P \tau$ is the amount of energy conserved in the PCRs presented by Ravetz et al. [3], and clarifies the role in PCRs of the law of conservation of energy, which is obscured when considering solely the triplet fusion upconversion mechanism in explaining the observed outcome. 


\section{Conclusions}

The evidence so far that the product of light's power $P$ times its period $\tau$, i.e. $P \tau$, is the amount of energy conserved in light-matter interaction is provided by experiments examining the outcome from capacitors illuminated by light. As such, $P \tau$ plays a primary role in the analysis of light-matter interaction because it competes with photons with energy given by Planck's constant $h$ times light's frequency $v$. Indeed, both $P \tau$ and the photon are called into play when conservation of energy is considered in light-matter interaction. However, as the energy $P \tau$ and the energy of the photon $\mathrm{h} v$ are different in the orders of magnitude, it appears necessary to shed light on which one of the two energies truly satisfies the energy balance in light-matter interaction.

Our research shows results clearly in favor of $P \tau$. Indeed, from the study of the photothermoelectric effect and of photoredox catalysis reactions, we infer that the hypothesis that $P \tau$ represents the amount of energy conserved in light-matter interaction is generally true. In addition, through $P \tau$, in the case of the photothermoelectric effect, we unveil that the size of the light's beam crosssectional area impinging on the photodetectors plays a major role in defining the performance of the photodetectors. With our analysis, the photodetector responsivities actually turn out to be higher than those reported in the original article. In the case of the photoredox catalysis reactions, we find that the magnitude of $P \tau$ involved in successful photoredox catalysis reactions is independent of the type of light used, whether near-infrared or blue. In addition, the involvement of $P \tau$ in the description of photoredox catalysis reactions helps to clarify the role of the law of conservation of energy, which was neglected by the authors of the original article.

The hypothesis that $P \tau$ represents the amount of energy conserved in lightmatter interaction was revealed to be also effective in the interaction of light with capacitors [1], field effect transistors [7] [8] [9], and, through our analysis, in the photothermoelectric effect excited by visible light and microwaves [2] [5]. We were able to reach the same conclusion in the investigation of infrared spectroscopy [10] and in vision in vertebrates [11]. The validity of this conclusion on $P \tau$ as the energy conserved in light-matter interaction with radio waves, microwaves, $\mathrm{X}$-rays and $\gamma$ rays still needs to be ascertained. Finally, the relationship between $P \tau$ and photocurrent formation also needs to be investigated. Assessing that $P \tau$ is the amount of energy conserved in light-matter interaction impacts: 1) the quantitative analysis of light-matter interaction; 2) the determination of the effectiveness of devices and phenomena based on light-matter interaction; and 3) the establishment of the conditions to obtain similar effects with different wavelengths.

\section{Acknowledgements}

The author thanks the Department of Physics and Astronomy of the James Madison University for supporting the research that results in this article. 


\section{Conflicts of Interest}

The author declares no conflicts of interest regarding the publication of this paper.

\section{References}

[1] Boone, D.E., Jackson, C.H., Swecker, A.T., Hergenrather, J.S., Wenger, K.S., Kokhan, O., Terzić, B., Melnikov, I., Ivanov, I.N., Stevens, E.C. and Scarel, G. (2018) Probing the Wave Nature of Light-Matter Interaction. World Journal of Condensed Matter Physics, 8, 62-89. https://doi.org/10.4236/wjcmp.2018.82005

[2] Lu, X., Liang, P. and Bao, X. (2019) Phonon-Enhanced Photothermoelectric Effect in $\mathrm{SrTiO}_{3}$ Ultra-Broad-Band Photodetector. Nature Communications, 10, 138. https://doi.org/10.1038/s41467-018-07860-0

[3] Ravetz, B.D., Pun, A.B., Churchill, E.M., Congreve, D.N., Rovis, T. and Campos, L.M. (2019) Photoredox Catalysis using Infrared Light via Triplet Fusion Upconversion. Nature, 565, 343-346. https://doi.org/10.1038/s41586-018-0835-2

[4] Vincent-Johnson, A.J., Vasquez, K.A., Bridstrup, J.E., Masters, A.E., Hu, X. and Scarel, G. (2011) Heat Recovery Mechanism in the Excitation of Radiative Polaritons by Broadband Infrared Radiation in Thin Oxide Films. Applied Physics Letters, 99, Article ID: 131901. https://doi.org/10.1063/1.3643464

[5] Skoblin, G., Sun, J. and Yurgens, A. (2018) Graphene Bolometer with Thermoelectric Readout and Capacitive Coupling to an Antenna. Applied Physics Letters, 112, Article ID: 063501. https://doi.org/10.1063/1.5009629

[6] Cabré, G., Garrido-Charles, A., Moreno, M., Bosch, M., Porta-de-la-Riva, M., Krieg, M., Gascón-Moya, M., Camarero, N., Gelabert, R., Lluch, J.M., Busqué, F., Hernando, J., Gorostiza, P. and Alibés, R. (2019) Rationally Designed Azobenzene Photoswitches for Efficient Two-Photon Neuronal Excitation. Nature Communications, 10, 907. https://doi.org/10.1038/s41467-019-08796-9

[7] Barati, F., Grossnickle, M., Su, S., Lake, R.L., Aji, V. and Gabor, N.M. (2017) Hot Carrier-Enhanced Interlayer Electron-Hole Pair Multiplication in 2D Semiconductor Heterostructure Photocells. Nature Nanotechnology, 12, 1134-1139. https://doi.org/10.1038/nnano.2017.203

[8] Adinolfi, V. and Sargent, E.H. (2017) Photovoltage Field-Effect Transistors. Nature, 542, 324-327. https://doi.org/10.1038/nature21050

[9] Sarker, B.K., Cazalas, E., Chung, T.-F., Childres, I., Jovanovic, I. and Chen, Y.P. (2017) Position-Dependent and Millimeter-Range Photodetection in Phototransistors with Micrometer-Scale Graphene on SiC. Nature Nanotechnology, 12, 668-674. https://doi.org/10.1038/nnano.2017.46

[10] Scarel, G. and Stevens, E.C. (2019) The Effect of Infrared Light's Power on the Infrared Spectra of Thin Films. World Journal of Condensed Matter Physics, 9, 1-21. https://doi.org/10.4236/wjcmp.2019.91001

[11] Scarel, G. (2019) Quantum and Non-Quantum Formulation of Eye's Adaptation to Light's Intensity Increments. World Journal of Condensed Matter Physics, 9, 62-74. https://doi.org/10.4236/wjcmp.2019.93005 